\title{
Patrizia Cavalli, Vita Meravigliosa
}

Torino, Einaudi, 2020, pp. 114, € 11

"I quattro firmatari si son perciò messi a leggere qualche riga, financo qualche pagina di Morante. Ciò è bastato loro per dire che Morante è scrittrice reazionaria, una bamboleggiante nipotina di De Amicis" (idem). Più che ad una precisa disamina critica legata a proposte sulla teoria del romanzo, il caso letterario alza un nebuloso polverone su quale metafisica della storia, in seno agli anni Settanta, sposare. Da una parte la linea marxista denuncia la rappresentazione di una mancata lotta di classe, "un'elegia della rassegnazione". Contrariamente, secondo Ginzburg, Morante "sceglie e raggiunge alcune fra le più sperdute creature della terra, segue il corso del loro destino e ne illumina la qualità misteriosa [...] la felicità e la sventura, la vita e la morte, risplendono di luce diversa, ma è sempre luce (Ginzburg, 1974).

Come ha di recente sottolineato Angela Borghesi, in un ponderoso e documentato studio, il 1974 è stato l'anno de La storia, il romanzo morantiano pubblicato a giugno per la collana Struzzi dell'editore Einaudi, in una tiratura di centomila copie, climaterico di una stagione di transizione letteraria. Fin da subito il romanzo si configura come uno spartiacque che segmenta la critica in due fronti fortemente polarizzati: da una parte $i$ tonitruanti plausi di Ginzburg e Garboli - fra i vari - e dall'altra gli strali veleniferi lanciati "con professionale disinvoltura" (Borghesi, 2018, p. 106) da Nanni Balestrini, Elisabetta Rasy, Letizia Paolozzi e Umberto Silva.

Una risposta simbolica al dibattito sul legame tra letteratura e storia, condotto a suon di elzeviri e recensioni, avviene pochi mesi dopo con l'esordio poetico di Patrizia Cavalli che sotto l'impulso della maestra - sì, proprio lei - Elsa Morante, pubblica per la collana Bianca Einaudi Le mie poesie non cambieranno il mondo. Il componimento proemiale risuona a chiare lettere, quasi nel tentativo di mettere a tacere la bagarre: "qualcuno mi ha detto / che certo le mie poesie / non cambieranno il mondo. / Io rispondo che certo sì / le mie poesie / non cambieranno il mondo". Da quel debutto luminoso e aurorale, la produzione di Patrizia Cavalli si è espansa, con parsimonia, pubblicando quasi sempre con cadenza settennale i suoi libri, oltre all'eccezione costituita dai due poemetti La guardiana e La patria, editi per Nottetempo. Vita meravigliosa è un libro che gioca tra il declamatorio e l'antifrastico in rapporto alla sfera semantica aperta dal primo settenario della 
quartina proemiale scelto come titolo: "vita meravigliosa / sempre mi meravigli / che pure senza figli / mi resti ancora sposa." (Cavalli, 2020, p. 5). Il libro è inaugurato da un bagliore penniano che invoca, quasi a ricalcare un antico inno omerico, una vita meravigliosa che le resta sposa senza figli. In vero controluce si nota come, il candido tenore glorificante, sia un costante puntellare le rovine a cui è sottoposta l'esistenza nei suoi ultimi rivoli. Per questa ragione la raccolta si configura come un'opulenta gerla riempita da Cavalli con tutto l'insieme di costellazioni tematiche e relative connotazioni retorico-stilistiche presenti in tutta la sua opera.

Se il secondo componimento, con dovizia endecasillabica richiama la sfera tematica del cielo, già esposta nell'omonima raccolta edita nel 1980, nelle sei sezioni che compongono la raccolta, ritornano le celebri porte: "io l'aspettavo fuori dalla porta / ma era così assorta nella sua ascesa / quasi rinocerontica mortale (idem, p. 7). Negando come di consueto, l'idea che all'essere umano possa essere affibbiato il concetto di identità - in senso aristotelico - "e io a chi parlo quando parlo da sola? Parlo a qualcuno che non sono io / ma una platea vista di sbieco al volo, / mutevole a seconda del mio tono" (ibidem, p. 39), l'autrice decide di far entrare una serie di oggetti consueti della contemporaneità, come fluoxetina, whiskey, mitigando l'ampio ricorso della tradizione storico-formale del sonetto con l'adozione di alcuni neologismi lessicali, come il verbo amusare. Se la "vita meravigliosa", non sa quale sarà l'approdo finale, Cavalli tenta una riconfigurazione dell'Eden: "lo preferisco falso e permanente dove la legge la decido io. / Abolirò memoria e nostalgia, / non ci sarà immaginazione ma un'aria mite e ferma che acconsente". (ibidem, p. 8). Un paradiso anomalo, forse immanente, che ricorda "la quiete altissima" de Il cantico del gallo silvestre di Leopardi. In questi termini, affinché la vita cavalliana meriti ancora l'aggettivazione di "meravigliosa" il grund del mistero deve rimanere in piena intermittenza, in una oscura condizione: "ma prima di morire / forse potrò capire / la mia incerta e oscura condizione. / Forse per non morire / continuo a non capire / sicura in questa chiara confusione" (ibidem, p. 77).

\title{
Riferimenti bibliografici
}

Borghesi, A. 2028. L'anno della Storia (1974-1975). Macerata: Quodlibet. Cavalli, P. 2020. Vita meravigliosa, Torino: Einaudi, 2020. Ginzburg, N. 1974. Vita immaginaria. Milano: Mondadori.

\author{
Pietro Polverini \\ Università di Macerata
}

